\title{
Age-Related Hypercholesterolemia and HMG-CoA Reductase Dysregulation: Sex Does Matter (A Gender Perspective)
}

\author{
Laura Trapani and Valentina Pallottini \\ Department of Biology, University of Roma Tre, Viale Marconi, 446, 00146 Rome, Italy \\ Correspondence should be addressed to Valentina Pallottini, vpallott@uniroma3.it
}

Received 29 October 2009; Revised 26 January 2010; Accepted 18 February 2010

Academic Editor: Jacek Witkowski

Copyright $\odot 2010$ L. Trapani and V. Pallottini. This is an open access article distributed under the Creative Commons Attribution License, which permits unrestricted use, distribution, and reproduction in any medium, provided the original work is properly cited.

\begin{abstract}
Although cardiovascular diseases are less prevalent in premenopausal women than in men, their occurrence in women increases at the onset of menopause, and the loss of female sex hormones contributes to the striking increase in cardiovascular morbidity and mortality in postmenopausal women. We present here a description of age-related disruption of lipid homeostasis, which particularly affects 3-hydroxy 3-methylglutaryl Coenzyme A reductase, the key rate-limiting enzyme in the cholesterol biosynthetic pathway. We further discuss the age- and gender-related dysregulation of this enzyme, providing new evidence for the different mechanisms driving dyslipidemia in elderly men and women. In addition, we introduce pharmacological methods of regulating HMGR and maintaining cholesterol homeostasis.
\end{abstract}

\section{Introduction}

Aging has been defined as the series of the deteriorative changes occurring during the adult period of life that underlie increased vulnerability to challenges and decreased survival [1]. This deterioration is responsible for both the commonly recognized sequential changes that accompany advancing age and the progressive increase in the chance of disease and death and is usually manifested as a progressive decrease in physiological functions.

Aging is characterized by the loss of homeostasis [2] that leads to changes in the biochemical composition of tissues [3-5], reduced ability to respond adaptively to environmental stimuli [6], and increased susceptibility and vulnerability to diseases [7] including coronary artery diseases $(\mathrm{CAD})$. The term CAD refers to pathologic changes within the coronary artery walls that result in diminished blood flow through these vessels. CAD can cause myocardial ischemia and possibly lead to acute myocardial infarction through three mechanisms - profound vascular spasm of the coronary arteries, formation of atherosclerotic plaques, and thromboembolism.

Although it is widely accepted that abnormal levels of lipids and/or lipoproteins in blood are modifiable risk factors for CAD $[8,9]$, the importance of lipid levels as prognostic factors in older adults is controversial. Several studies have suggested that the association between cholesterol concentration and atherosclerotic CAD weakens with age, and that screening and treating older adults for dyslipidemia provides little potential benefit $[10,11]$. In contrast, other reports suggest that lipoprotein levels remain a significant risk factor for CAD in the elderly and that treatment of dyslipidemia in the elderly may have a greater impact on CAD mortality than in younger people because the total attributable risk from dyslipidemia is greater in the older age group $[12,13]$.

The mechanisms behind this age-related dyslipidemia are incompletely characterized. Some evidence demonstrates that the causes of age-related disruption of lipid homeostasis include the gradual decline in fractional clearance of LDL with increasing age, the progressively reduced ability to remove cholesterol through conversion to bile acids, and the decreased activity of the rate-limiting enzyme in bile acid biosynthesis, cholesterol $7 \alpha$-hydroxylase $(\mathrm{C} 7 \alpha \mathrm{OH})$. Moreover, an interesting hypothesis states that critical changes in cholesterol and lipoprotein metabolism depend on the progressive decrease in growth hormone (GH) secretion, a characteristic feature of aging. GH plays an important role in cholesterol homeostasis by either modulating the expression of hepatic LDLr [14] or controlling the activity of cholesterol $7 \alpha$-hydroxylase [15]. 


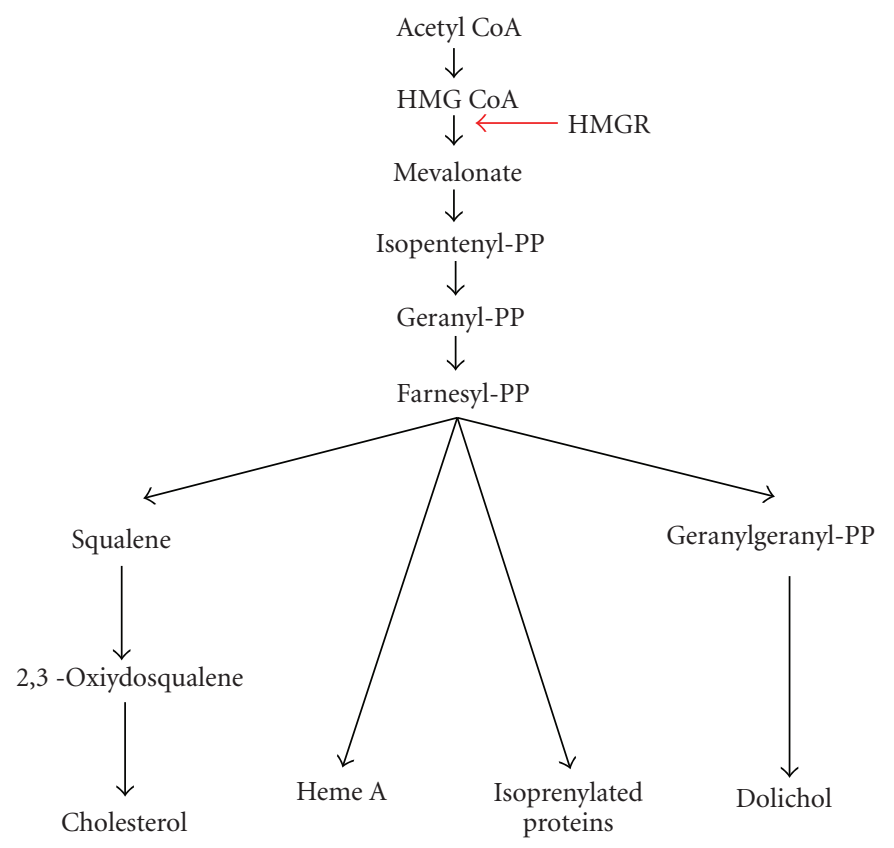

FIGURE 1: Schematic illustration of the biosynthetic pathway of HMGR end-products.

$\mathrm{CAD}$ is less prevalent in premenopausal women than in men, but its occurrence in women increases at the onset of menopause, and the loss of female sex hormones contributes to the striking increase in the incidence of cardiovascular morbidity and mortality in postmenopausal women. Estrogen replacement therapy results in improved lipoprotein profiles in postmenopausal women, but these improved profiles account for less than half of the cardioprotective effects of estrogen replacement therapy. The cardioprotective effects of estrogens include beneficial changes in plasma lipid levels (increased high-density lipoprotein (HDL), decreased total cholesterol and low-density lipoprotein (LDL), and decreased LDL oxidation) [16, 17], antiplatelet and antioxidant effects, and preservation of endothelium-mediated vasodilation $[18,19]$. Providing further evidence for the cardioprotective role of estrogen, studies performed in estrogendeficient animal models have demonstrated a disruption of lipid homeostasis [20].

Cholesterol plays an essential role in the synthesis of new membranes, the turnover of lipids in existing membranes, and the biosynthesis of products such as steroid hormones and bile acids [21]. Cholesterol homeostasis is maintained by a feedback regulatory system that senses the level of cholesterol in cell membranes and modulates both the transcription of genes encoding proteins involved in cholesterol biosynthesis and posttranscriptional events along with the uptake of cholesterol from plasma lipoproteins [22]. Maintenance of cholesterol homeostasis is regulated by both the receptor-mediated endocytosis of LDL by LDL receptors (LDLr) and de novo cholesterol synthesis via the rate-limiting enzyme 3-hydroxy-3-methylglutaryl coenzyme A reductase (HMGR) [23]. Because of the pivotal role of HMGR in cholesterol and nonsterol isoprenoid compound biosynthesis, most of the mechanisms controlling cholesterol homeostasis are related to short- and long-term regulation of HMGR.

To provide new evidence for the different mechanisms driving dyslipidemia in elderly men and women, this review will focus on age-related disruption of lipid homeostasis, and in particular on the age- and gender-related dysregulation of HMGR, the key rate-limiting enzyme in the cholesterol biosynthetic pathway.

\section{HMGR Regulation in Adults}

Cholesterol biosynthesis occurs through a tightly regulated pathway that employs multiple feedback mechanisms to maintain homeostasis [24]. Over the past several decades, much work has focused on the regulation of HMGR, which catalyzes the conversion of HMG-CoA to mevalonate (MVA) through a four-electron oxidoreduction. This reaction is the rate-limiting step in the synthesis of cholesterol and other isoprenoids such as dolichol, isopentenyladenine, which is present in some tRNAs, heme A, ubiquinone, and prenylated proteins such as Ras and Rab proteins (Figure 1) [24].

Encoded by the HMGR gene located on chromosome 5 of human genome, HMGR consists of a single 888 amino acid polypeptide chain. The $\mathrm{N}$-terminal membrane domain contains 339 hydrophobic residues that span the endoplasmic reticulum (ER) membrane and contains the sterol-sensing domain (SSD), which is responsible for the binding of sterols and other MVA derivatives that accelerate enzyme degradation [25], while the catalytic site is located in the hydro-soluble C-terminal cytoplasmic domain. A linker region (residues 340-459) connects these two portions of the protein [2]. 
Short-term regulation of HMGR is achieved through its phosphorylation and dephosphorylation, both of which can affect enzyme activity. Phosphorylation of residue S872 of HMGR decreases its catalytic activity, and removal of this phosphate results in reactivation [26, 27]. AMP-activated kinase (AMPK) appears to be the major HMGR kinase in the liver, where cholesterologenesis takes place. AMPK is a heterotrimeric serine/threonine kinase consisting of a catalytic $\alpha$ subunit and regulatory $\beta$ and $\gamma$ subunits [28]. AMPK is activated by phosphorylation of the $\alpha$ subunit at a specific threonine residue (Thr172) [29]. HMGR activation is mediated by its dephosphorylation by protein phosphatase 2A (PP2A), which regulates a significant network of cellular events [30].

In addition to this short-term regulation, HMGR is subject to transcriptional, translational, and posttranslational control [31]. These levels of control, which are mediated by changes in intracellular sterol levels and cholesterol uptake by LDLr, can result in changes of over 200-fold in HMGR levels [32]. Both LDLr and HMGR are produced in response to activation of the Sterol Regulatory Element Binding Proteins (SREBPs), and particularly SREBP-2, in the liver [33, 34].

Long-term regulation of HMGR is mediated by a pair of membrane-bound proteins, SREBP cleavage activating protein (Scap) and Insulin-induced gene (Insig), which directly bind sterols and thereby sense sterol concentration in the membranes of the ER. As a result of these binding events, both Scap and Insig undergo conformational changes that initiate a series of molecular events blocking Scap's ability to transport SREBPs to the Golgi, terminating cholesterol synthesis and uptake [32]. Furthermore, the intracellular accumulation of sterols induces HMGR to bind Insig, promoting ubiquitination and proteasomal degradation of HMGR [35].

Several hormones, including insulin, glucagon, glucocorticoids, thyroid hormone, and estrogen, regulate the expression of hepatic HMGR in animals. Insulin likely stimulates HMGR expression by increasing its rate of transcription, while glucagon opposes this effect. Hepatic HMGR activity undergoes significant diurnal variations due to changes in the levels of immunoreactive proteins, which are primarily mediated by changes in insulin and glucagon levels. Thyroid hormone increases hepatic HMGR levels by acting to increase both transcription and mRNA stability, while glucocorticoids decrease hepatic HMGR expression by destabilizing HMGR mRNA [36]. The effects of estrogen on HMGR expression are still debated. Some studies suggest that estrogens act to increase hepatic HMGR activity primarily by stabilizing HMGR mRNA and that deficiencies in those hormones that act to increase hepatic HMGR gene expression lead to elevated serum cholesterol levels [36]. On the other hand, studies using the DLD1 cell line suggest that estrogens induce an early increase in LDLr at both the mRNA and the protein level and later cause decreases in HMGR activity and protein expression [37].

Although the mechanisms that regulate cholesterol homeostasis are well known, [22] the literature describing putative physiological sex differences in cholesterol homeostasis-related proteins is limited [38-42]. Further- more, most of these papers are fragmented and very old, and none of them focus on the mechanisms underlying these sex-related differences. De Marinis and coworkers [43] provided evidence of sex-related physiological differences in proteins involved in cholesterol homeostasis. In particular, activity and expression levels of HMGR are lower in 3month-old female rats and in 17- $\beta$-estradiol-treated 3month-old male rats than in 3-month-old untreated male rats. Moreover, 3-month-old female rats express lower levels of SREBP-2 and higher levels of Insig than their male counterparts. Sex-related variations in expression of these regulatory proteins are functionally consistent with the wellaccepted classical model of HMGR behavior [22, 32], and no sex-related differences have been observed in either LDLr expression or cholesterol levels, excluding the involvement of end-product feedback in presence of physiological content of estradiol. The difference in the expression pattern of regulatory proteins in males and females seems to be related to the presence of estrogen, and altered expression of these regulatory proteins drives the sex-related differences in HMGR expression.

\subsection{Sex-Related Differences in HMGR Dysregulation during} Aging. Due to the serious health-related consequences of aging, significant efforts have been made to provide a more complete understanding of this particular stage of life. Current research aiming to delineate the biological mechanisms of aging has yielded valuable information about the molecular basis of age-related physiological deterioration. One of the critical problems associated with aging is the increased incidence of $\mathrm{CAD}$ and, more generally, cardiovascular diseases (CVD). Many risk factors predispose elderly people to develop pathologies related to failure of the heart vasculature, including hypercholesterolemia. Thus, understanding the mechanisms driving increased cellular and plasma cholesterol content during aging is essential in defining specific intervention points.

During aging, hepatic lipid modifications occur. In particular, studies of 24-month-old male rats showed increased plasma cholesterol levels and increased hepatic cholesterol synthesis accompanied by full activation of HMGR [44, 45], which was dependent on the well-known age-related increase in reactive oxygen species (ROS) [46, 47]. The agerelated increase in activation of HMGR has been associated with an increase in ROS $[48,49]$. The current model proposes that increased ROS levels result in activation of both p38 and AMPK $\alpha$. In turn, p38 activation may result in an increase in association of PP2A with HMGR, leading to dephosphorylation and increased activation of HMGR. AMPK $\alpha$ kinase activity is impaired by the enhanced association of PP2A with HMGR [50]. Moreover, findings in $\mathrm{H}_{2} \mathrm{O}_{2}$-stimulated HepG2 cells demonstrate that the effect of ROS on HMGR dephosphorylation is mediated by activation of the p38/MAPK pathway [45].

In addition to the short-term regulation mediated by phosphorylation and dephosphorylation, long-term regulation of HMGR also appears to be affected by aging. Age-related variations in hormone levels and hormone 
sensitivity induce a decreased ability to maintain homeostatic potential, and these hormonal changes are always associated with changes in the expression or functionality of some molecules. In particular, it has been clearly demonstrated that the age-related decrease in insulin sensitivity induces changes in some factors involved in cholesterol metabolism, such as Insig-1 protein. This age-related reduction in Insig expression results in slower degradation of HMGR [51, 52].

While many studies have established that susceptibility to $\mathrm{CAD}$ increases with age, little is known about the mechanisms underlying the increased incidence of CAD in postmenopausal women as compared to men of the same age.

Previous studies have shown that 12-month-old estropausal rats, in which estrogen levels are decreased, have higher levels of plasma cholesterol, increased activation of HMGR, and decreased LDLr membrane exposure than 3-month-old female rats. These changes result in decreased cholesterol uptake and increased cholesterol synthesis, supporting the correlation between hypercholesterolemia, aging, and estropause. Increased activation of HMGR does not depend on an increase in ROS as seen in aged-matched male rats [53]. Instead, HMGR activation seems to be due to decreased activation of AMPK during the period of 17- $\beta$ estradiol deficiency that occurs at the beginning of estropause; this decrease in AMPK activation results in decreased phosphorylation of HMGR.

Treatment of older female rats with $17-\beta$ estradiol results in restoration of normal cholesterol levels, decreased activation of HMGR, and increased LDLr exposure on the plasma membrane. Furthermore, while $17-\beta$ estradiol treatment does not fully restore AMPK activation, AMPK is sufficiently activated in older $17-\beta$ estradiol-treated female rats to phosphorylate HMGR, reestablishing HMGR activity [54]. This estradiol-induced enhancement in AMPK activation is supported by studies by Schulz and coworkers, who demonstrated that estradiol-mediated AMPK activation was independent of estrogen receptor ligand engagement and involved catechol metabolism of estradiol [55].

The decrease in estradiol levels that occurs at the onset of estropause does not affect long-term regulation of HMGR, but mediates short-term HMGR regulation by controlling activation of AMPK. Thus, a relationship exists between changes in estrogen levels and HMGR-related modulation of cholesterolemia in older female rats. The protective role played by estrogens in modulating the lipid profile is mediated not only through increases in plasma HDL, decreases in plasma LDL, and decreased oxidation, but also through regulation of AMPK activation, which inhibits HMGR and cholesterol synthesis.

\section{Conclusion and Future Perspectives}

In elderly men and women, HMGR is highly activated; however, the mechanisms driving dysregulation of HMGR appear to be gender-dependent. Studies of aged male rats suggest that in males, HMGR dysregulation is due to increased association between PP2A and HMGR, which results in increased activation of HMGR. On the other hand, studies of estropausal female rats, in which estrogen levels are decreased, suggest that the menopause-related increase in HMGR activity is caused by the decreased activation of AMPK observed during estrogen deficiency.

The regulation of HMGR activity has been an attractive target for pharmacological treatment of hypercholesterolemia, the main risk factor for CAD. Consequently, better understanding of the molecular mechanisms that drive dysregulation of HMGR activity and hypercholesterolemia in aged men and women could provide gender-specific targets for treatments to lower plasma cholesterol content, resulting in both prevention and reduction of one of the main risk factors for cardiovascular diseases.

Decreased cellular cholesterol synthesis leads to a homeostatic response involving up-regulation of cell-surface receptors that bind atherogenic lipoproteins such as LDL and VLDL. These lipoproteins are taken up by the cell and degraded [56], resulting in a reduction in circulating atherogenic lipoproteins. This process helps to explain the clinical usefulness of HMGR inhibitors (statins).

HMGR inhibition results in not only reductions in cellular and plasma cholesterol levels, but also reductions in other products synthesized through the cholesterol biosynthetic pathway, such as ubiquinone, prenylated proteins, and dolichol. The restoration of HMGR to its normal activation state regulates the physiological synthesis of cholesterol within cells, and restored cellular cholesterol levels are in turn reflected in proper membrane LDLr presence [22].

Statins are effective means of primary and secondary prevention of ischemic heart disease (IHD) in middle-aged men; however, proof of the efficacy of statins in preventing development and progression of IHD in women and elderly people is less convincing. In the PROSPER (Prospective Study of Pravastatin in the Elderly at Risk of vascular disease) trial, pravastatin not only had no effect in men and women aged 70-82 years, but also significantly increased the rate of breast cancer in these patients. In the ALLHAT-LLT (Antihypertensive and Lipid-Lowering Treatment to Prevent Heart Attack Trial) trial, pravastatin lowered neither the total number of nonfatal myocardial infarctions and IHD deaths nor total mortality in patients aged 65 years and older and in women [57].

Considering the undesirable side effects of statins and the effects of these drugs on other important compounds in addition to cholesterol, development of new pharmacologically active compounds capable of regulating plasma cholesterol content is critical to effectively control this important CVD risk factor.

Many recent studies have described new compounds able to decrease plasma cholesterol content. Although the exact mechanisms by which these compounds act are unknown, some of these compounds affect expression levels and activity of HMGR. These novel compounds could thus represent the future of hypercholesterolemia therapy and should be studied further.

Hypercholesterolemia can also be approached using other therapeutic targets; for example, proprotein convertase subtilisin/kexin type 9 (PCSK9) has been implicated as 
an important regulator of LDL metabolism. PCSK9 belongs to the subtilisin family of serine proteases and is highly expressed in the liver [58]. Secreted PCSK9 modulates LDL levels through posttranslational down-regulation of hepatic LDLr protein [34]. Down-regulation of PCSK9 could thus be effective in decreasing plasma cholesterol content by increasing LDLr levels without affecting activity of HMGR and its end-products.

In addition, squalene synthase, an enzyme that is downstream of HMGR in the cholesterol synthesis pathway, modulates the first committed step of hepatic cholesterol biosynthesis at the final branching point of the cholesterol biosynthetic pathway. Pharmacologic inhibitors of squalene epoxidase and oxidosqualene cyclase, two enzymes that act downstream of squalene synthase, may thus be useful in reducing plasma LDL content [59].

Moreover, the identification of Scap and Insig as sterolbinding proteins in mammalian cells has added a new level of molecular detail to the understanding of regulation of the SREBP pathway and subsequent regulation of HMGR levels, providing new potential targets for pharmacological intervention.

Much work remains to define the relationship between hormonal changes and their effects on transcription factors and cholesterol metabolism in different physiological and pathological conditions. For example, some studies suggest that estrogens are able to regulate cholesterol homeostasis without directly affecting HMGR [43]. Additionally, more detailed studies are required to define the specific roles of Insig proteins and to determine the metabolic consequences of their reciprocal regulation. In fact, since they are required for feedback regulation of SREBP processing and HMGR degradation, Insigs may represent a new target for pharmacological intervention to maintain blood cholesterol levels within the optimal range. None of the papers we have cited focus on the sex-related differences.

Thus, although HMGR plays a pivotal role in regulating cholesterol metabolism, future studies should address sexrelated differences in the cholesterol biosynthetic pathway to identify new targets for customized pharmacological treatment of hypercholesterolemia.

\section{Acknowledgments}

The authors wish to thank past and present members of their laboratories who contributed with data and discussions to the ideas presented here. In particular, the invaluable and dedicated work of our mentor Professor Anna Trentalance is warmly acknowledged.

\section{References}

[1] E. J. Masoro, "Overview of caloric restriction and ageing," Mechanisms of Ageing and Development, vol. 126, no. 9, pp. 913-922, 2005.

[2] R. G. Cutler, "The dysdifferentiative hypothesis of mammalian ageing and longevity," in The Ageing Brain, E. Jacobini, et al., Ed., Raven Press, New York, NY, USA, 1982.
[3] B. L. Strehler, Time, Cells, and Ageing, Academic Press, New York, NY, USA, 1977.

[4] J. Bjorksten, "Cross linkage and the ageing process," in Theoretical Aspects of Ageing, M. Rothstein, Ed., Academic Press, New York, NY, USA, 1974.

[5] R. R. Kohn, "Ageing of animals: possible mechanisms," in Principles of Mammalian Ageing, R. R. Kohn, Ed., PrenticeHall, Englewood Cliffs, NJ, USA, 1978.

[6] R. C. Adelman, G. W. Britton, S. Rotenberg, L. Ceci, and K. Karoly, "Endocrine regulation of enzyme activity in aging animals of different genotypes," Birth Defects: Original Article Series, vol. 14, no. 1, pp. 355-364, 1978.

[7] J. A. Brody and D. B. Brock, "Epidemiological and statistical characteristics of the United States elderly population," in Handbook of the Biology of Ageing, C. E. Finch and E. L. Schneider, Eds., Van Nostrand Reinhold, New York, NY, USA, 1985.

[8] W. H. Ettinger Jr., P. W. Wahl, L. H. Kuller, et al., "Lipoprotein lipids in older people: results from the Cardiovascular Health Study. The CHS Collaborative Research Group," Circulation, vol. 86, no. 3, pp. 858-869, 1992.

[9] W. P. Castelli, "Epidemiology of coronary heart disease: the Framingham study," American Journal of Medicine, vol. 76, no. 2A, pp. 4-12, 1984.

[10] A. M. Garber, B. Littenberg, H. C. Sox Jr., J. L. Wagner, and M. Gluck, "Costs and health consequences of cholesterol screening for asymptomatic older Americans," Archives of Internal Medicine, vol. 151, no. 6, pp. 1089-1095, 1991.

[11] R. Benfante and D. Reed, "Is elevated serum cholesterol level a risk factor for coronary heart disease in the elderly?" Journal of the American Medical Association, vol. 263, no. 3, pp. 393-396, 1990.

[12] E. Barrett-Connor, L. Suarez, K.-T. Khaw, M. H. Criqui, and D. L. Wingard, "Ischemic heart disease risk factors after age 50," Journal of Chronic Diseases, vol. 37, no. 12, pp. 903-908, 1984.

[13] W. P. Castelli, P. W. F. Wilson, D. Levy, and K. Anderson, "Cardiovascular risk factors in the elderly," American Journal of Cardiology, vol. 63, no. 16, pp. H12-H19, 1989.

[14] M. Matasconi, P. Parini, B. Angelin, and M. Rudling, "Pituitary control of cholesterol metabolism in normal and LDL receptor knock-out mice: effects of hypophysectomy and growth hormone treatment," Biochimica et Biophysica Acta, vol. 1736, no. 3, pp. 221-227, 2005.

[15] M. Rudling, P. Parini, and B. Angelin, "Growth hormone and bile acid synthesis. Key role for the activity of hepatic microsomal cholesterol 7alpha-hydroxylase in the rat," Journal of Clinical Investigation, vol. 99, no. 9, pp. 2239-2245, 1997.

[16] D. F. Skafar, R. Xu, J. Morales, J. Ram, and J. R. Sowers, "Female sex hormones and cardiovascular disease in women," Journal of Clinical Endocrinology and Metabolism, vol. 82, no. 12, pp. 3913-3918, 1997.

[17] K. Moorthy, U. C. S. Yadav, A. K. Mantha, et al., "Estradiol and progesterone treatments change the lipid profile in naturally menopausal rats from different age groups," Biogerontology, vol. 5, no. 6, pp. 411-419, 2004.

[18] S. R. J. Maxwell, "Women and heart disease," Basic Research in Cardiology, vol. 93, supplement 2, pp. 79-84, 1998.

[19] K. Moorthy, D. Sharma, S. F. Basir, and N. Z. Baquer, "Administration of estradiol and progesterone modulate the activities of antioxidant enzyme and aminotransferases in naturally menopausal rats," Experimental Gerontology, vol. 40, no. 4, pp. 295-302, 2005. 
[20] P. A. Heine, J. A. Taylor, G. A. Iwamoto, D. B. Lubahn, and P. S. Cooke, "Increased adipose tissue in male and female estrogen receptor-a knockout mice," Proceedings of the National Academy of Sciences of the United States of America, vol. 97, no. 23, pp. 12729-12734, 2000.

[21] C. Martini and V. Pallottini, "Cholesterol: from feeding to gene regulation," Genes and Nutrition, vol. 2, no. 2, pp. 181-193, 2007.

[22] M. S. Brown and J. L. Goldstein, "A proteolytic pathway that controls the cholesterol content of membranes, cells, and blood," Proceedings of the National Academy of Sciences of the United States of America, vol. 96, no. 20, pp. 11041-11048, 1999.

[23] P. J. Espenshade and A. L. Hughes, "Regulation of sterol synthesis in eukaryotes," Annual Review of Genetics, vol. 41, pp. 401-427, 2007.

[24] J. L. Goldstein and M. S. Brown, "Regulation of the mevalonate pathway," Nature, vol. 343, no. 6257, pp. 425-430, 1990.

[25] T. Ravid, R. Doolman, R. Avner, D. Harats, and J. Roitelman, "The ubiquitin-proteasome pathway mediates the regulated degradation of mammalian 3-hydroxy-3-methylglutarylcoenzyme A reductase," Journal of Biological Chemistry, vol. 275, no. 46, pp. 35840-35847, 2000.

[26] Z. H. Beg, J. A. Stonik, and H. B. Brewer Jr., "Phosphorylation of hepatic 3-hydroxy-3-methylglutaryl coenzyme A reductase and modulation of its enzymic activity by calcium-activated and phospholipid-dependent protein kinase," Journal of Biological Chemistry, vol. 260, no. 3, pp. 1682-1687, 1985.

[27] R. V. Omkumar, B. G. Darnay, and V. W. Rodwell, "Modulation of Syrian hamster 3-hydroxy-3-methylglutaryl-CoA reductase activity by phosphorylation. Role of serine 871 ," Journal of Biological Chemistry, vol. 269, no. 9, pp. 6810-6814, 1994.

[28] D. G. Hardie, S. A. Hawley, and J. W. Scott, "AMP-activated protein kinase-development of the energy sensor concept," Journal of Physiology, vol. 574, part 1, pp. 7-15, 2006.

[29] S. A. Hawley, M. Davison, A. Woods, et al., "Characterization of the AMP-activated protein kinase kinase from rat liver and identification of threonine 172 as the major site at which it phosphorylates AMP-activated protein kinase," Journal of Biological Chemistry, vol. 271, no. 44, pp. 27879-27887, 1996.

[30] V. Janssens and J. Goris, "Protein phosphatase 2A: a highly regulated family of serine/threonine phosphatases implicated in cell growth and signalling," Biochemical Journal, vol. 353, part 3, pp. 417-439, 2001.

[31] F. Xu, S. D. Rychnovsky, J. D. Belani, H. H. Hobbs, J. C. Cohen, and R. B. Rawson, "Dual roles for cholesterol in mammalian cells," Proceedings of the National Academy of Sciences of the United States of America, vol. 102, no. 41, pp. 14551-14556, 2005.

[32] J. L. Goldstein, R. A. DeBose-Boyd, and M. S. Brown, "Protein sensors for membrane sterols," Cell, vol. 124, no. 1, pp. 35-36, 2006.

[33] M. S. Brown and J. L. Goldstein, "The SREBP pathway: regulation of cholesterol metabolism by proteolysis of a membrane-bound transcription factor," Cell, vol. 89, no. 3, pp. 331-340, 1997.

[34] J. D. Horton, J. L. Goldstein, and M. S. Brown, "SREBPs: activators of the complete program of cholesterol and fatty acid synthesis in the liver," Journal of Clinical Investigation, vol. 109, no. 9, pp. 1125-1131, 2002.

[35] N. Sever, B.-L. Song, D. Yabe, J. L. Goldstein, M. S. Brown, and R. A. DeBose-Boyd, "Insig-dependent ubiquitination and degradation of mammalian 3-hydroxy-3-methylglutaryl-CoA reductase stimulated by sterols and geranylgeraniol," Journal of Biological Chemistry, vol. 278, no. 52, pp. 52479-52490, 2003.

[36] G. C. Ness and C. M. Chambers, "Feedback and hormonal regulation of hepatic 3-hydroxyl-3- methylglutaryl coenzyme A reductase: the concept of cholesterol buffering capacity," Proceedings of the Society for Experimental Biology and Medicine, vol. 224, no. 1, pp. 8-9, 2000.

[37] C. Messa, M. Notarnicola, F. Russo, et al., "Estrogenic regulation of cholesterol biosynthesis and cell growth in DLD-1 human colon cancer cells," Scandinavian Journal of Gastroenterology, vol. 40, no. 12, pp. 1454-1461, 2005.

[38] Y. J. Abul-Hajj, "Stimulation by estradiol benzoate of hepatic beta-hydroxy-beta-methylglutaryl coenzyme A reductase in normal and ovariectomized rats," Steroids, vol. 31, no. 6, pp. 841-847, 1978.

[39] S. E. Carlson, A. D. Mitchell, M. L. Carter, and S. Goldfarb, "Evidence that physiologic levels of circulating estrogens and neonatal sex-imprinting modify postpubertal hepatic microsomal 3-hydroxy-3-methylglutaryl coenzyme A reductase activity," Biochimica et Biophysica Acta, vol. 633, no. 2, pp. 154-161, 1980.

[40] Y.-S. Choi, S. Goto, I. Ikeda, and M. Sugano, "Age-related changes in lipid metabolism in rats: the consequence of moderate food restriction," Biochimica et Biophysica Acta, vol. 963, no. 2, pp. 237-242, 1988.

[41] K. N. Hewitt, W. C. Boon, Y. Murata, M. E. Jones, and E. R. Simpson, "The aromatase knockout mouse presents with a sexually dimorphic disruption to cholesterol homeostasis," Endocrinology, vol. 144, no. 9, pp. 3895-3903, 2003.

[42] M. Kojima and M. Degawa, "Gender-related difference in altered gene expression of a sterol regulatory element binding protein, SREBP-2, by lead nitrate in rats: correlation with development of hypercholesterolemia," Journal of Applied Toxicology, vol. 26, no. 4, pp. 381-384, 2006.

[43] E. De Marinis, C. Martini, A. Trentalance, and V. Pallottini, "Sex differences in hepatic regulation of cholesterol homeostasis," Journal of Endocrinology, vol. 198, no. 3, pp. 635-643, 2008.

[44] M. Marino, V. Pallottini, C. D’Eramo, G. Cavallini, E. Bergamini, and A. Trentalance, "Agerelated changes of cholesterol and dolichol biosynthesis in rat liver," Mechanisms of Ageing and Development, vol. 123, no. 8, pp. 1183-1189, 2002.

[45] V. Pallottini, C. Martini, G. Cavallini, et al., "Age-related HMG-CoA reductase deregulation depends on ROS-induced p38 activation," Mechanisms of Ageing and Development, vol. 128, no. 11-12, pp. 688-695, 2007.

[46] D. Harman, "Aging: a theory based on free radical and radiation chemistry," Journal of gerontology, vol. 11, no. 3, pp. 298-300, 1956.

[47] E. R. Stadtman, "Protein oxidation and ageing," Free Radical Research, vol. 40, no. 12, pp. 1250-1258, 2006.

[48] V. Pallottini, C. Martini, A. Pascolini, et al., "3-hydroxy-3methylglutaryl coenzyme A reductase deregulation and agerelated hypercholesterolemia: a new role for ROS," Mechanisms of Ageing and Development, vol. 126, no. 8, pp. 845-851, 2005.

[49] V. Pallottini, C. Martini, A. M. Bassi, P. Romano, G. Nanni, and A. Trentalance, "Rat HMG CoA reductase activation in thioacetamide-induced liver injury is related to an increased reactive oxygen species content," Journal of Hepatology, vol. 44, no. 2, pp. 368-374, 2006. 
[50] C. Martini, V. Pallottini, G. Cavallini, A. Donati, E. Bergamini, and A. Trentalance, "Caloric restrictions affect some factors involved in age-related hypercholesterolemia," Journal of Cellular Biochemistry, vol. 101, no. 1, pp. 235-243, 2007.

[51] V. Pallottini, L. Montanari, G. Cavallini, E. Bergamini, Z. Gori, and A. Trentalance, "Mechanisms underlying the impaired regulation of 3-hydroxy-3-methylglutaryl coenzyme A reductase in aged rat liver," Mechanisms of Ageing and Development, vol. 125, no. 9, pp. 633-639, 2004.

[52] V. Pallottini, C. Martini, G. Cavallini, et al., "Modified HMGCoA reductase and LDLr regulation is deeply involved in agerelated hypercholesterolemia," Journal of Cellular Biochemistry, vol. 98, no. 5, pp. 1044-1053, 2006.

[53] S. Straniero, G. Cavallini, A. Donati, et al., "Stimulation of autophagy by antilipolytic drugs may rescue rodents from ageassociated hypercholesterolemia," Rejuvenation Research, vol. 12, no. 2, pp. 77-84, 2009.

[54] L. Trapani, F. Violo, and V. Pallottini, "Hypercholesterolemia and 3-hydroxy-3-methylglutaryl coenzyme A reductase regulation in aged female rats," Experimental Gerontology, vol. 45, no. 2, pp. 119-128, 2010.

[55] E. Schulz, E. Anter, M. H. Zou, and J. F. Keaney Jr., "Estradiolmediated endothelial nitric oxide synthase association with heat shock protein 90 requires adenosine monophosphatedependent protein kinase," Circulation, vol. 111, no. 25, pp. 473480, 2005.

[56] M. S. Brown and J. L. Goldstein, "Cholesterol feedback: from Schoenheimers bottle to Scaps MELADL," Journal of Lipid Research, vol. 50, pp. S15-S27, 2009.

[57] D. V. Preobrazhenskil̆, B. A. Sidorenko, S. A. Pataraia, I. D. Vyshinskaia, and O. V. Borisenko, "Hypercholesterolemia in men and women of various age-part II: the problem of efficacy and safety of statins," Kardiologiia, vol. 47, no. 11, pp. 75-85, 2007.

[58] J. C. Y. Chan, D. E. Piper, Q. Cao, et al., "A proprotein convertase subtilisin/kexin type 9 neutralizing antibody reduces serum cholesterol in mice and nonhuman primates," Proceedings of the National Academy of Sciences of the United States of America, vol. 106, no. 24, pp. 9820-9825, 2009.

[59] S. Seiki and W. H. Frishman, "Pharmacologic inhibition of squalene synthase and other downstream enzymes of the cholesterol synthesis pathway: a new therapeutic approach to treatment of hypercholesterolemia," Cardiology in Review, vol. 17, no. 2, pp. 70-76, 2009. 


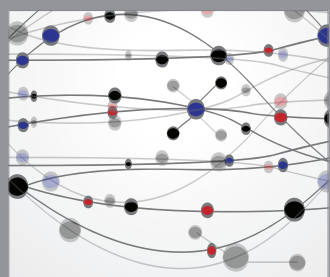

The Scientific World Journal
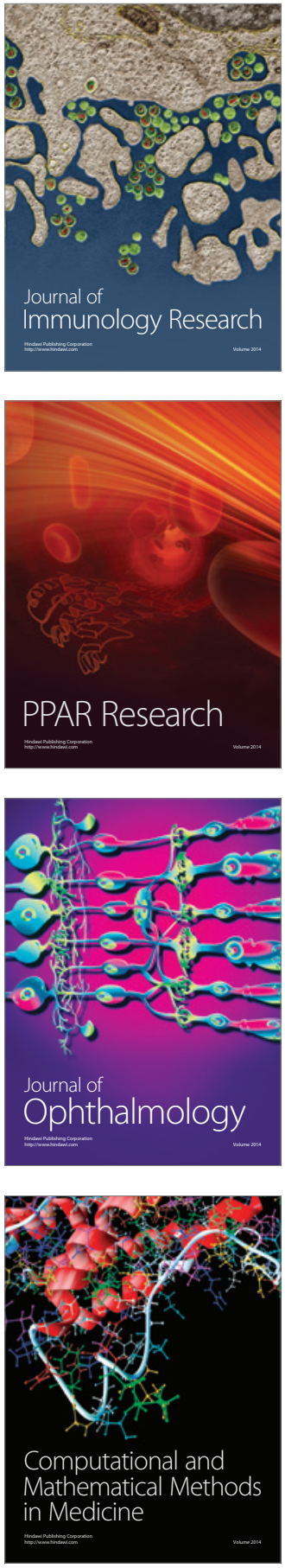

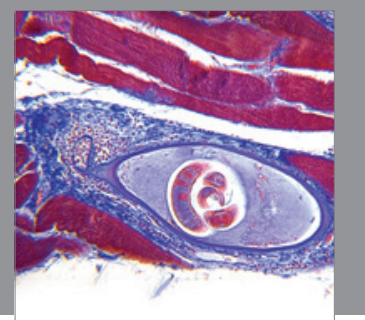

Gastroenterology

Research and Practice
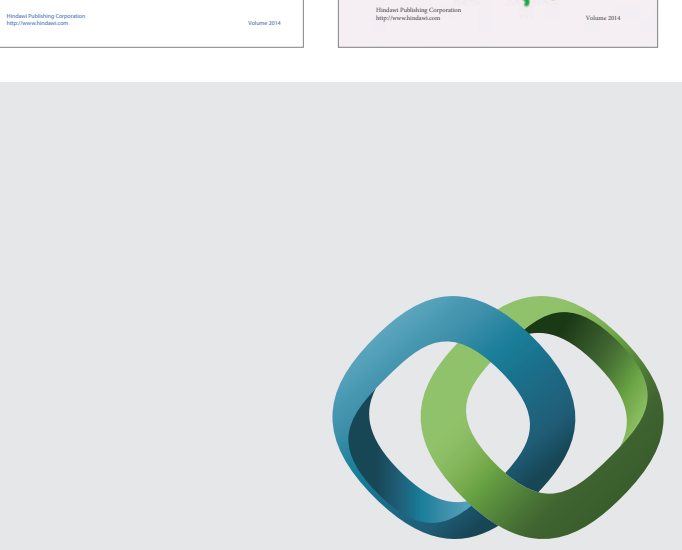

\section{Hindawi}

Submit your manuscripts at

http://www.hindawi.com
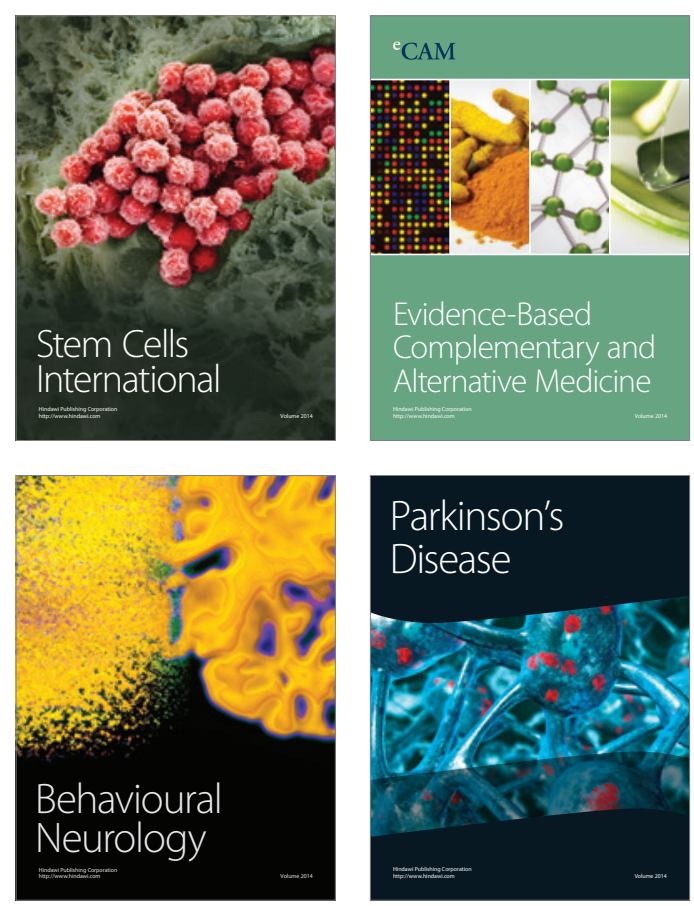

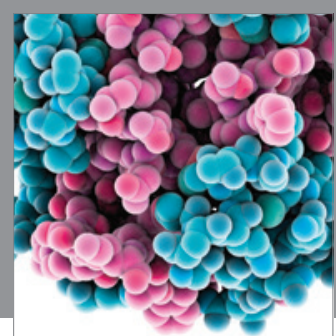

Journal of
Diabetes Research

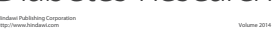

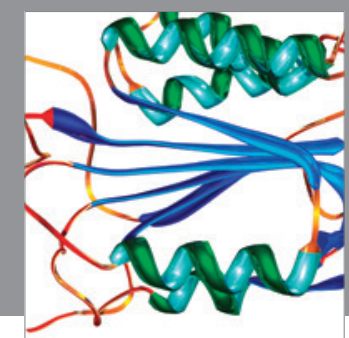

Disease Markers
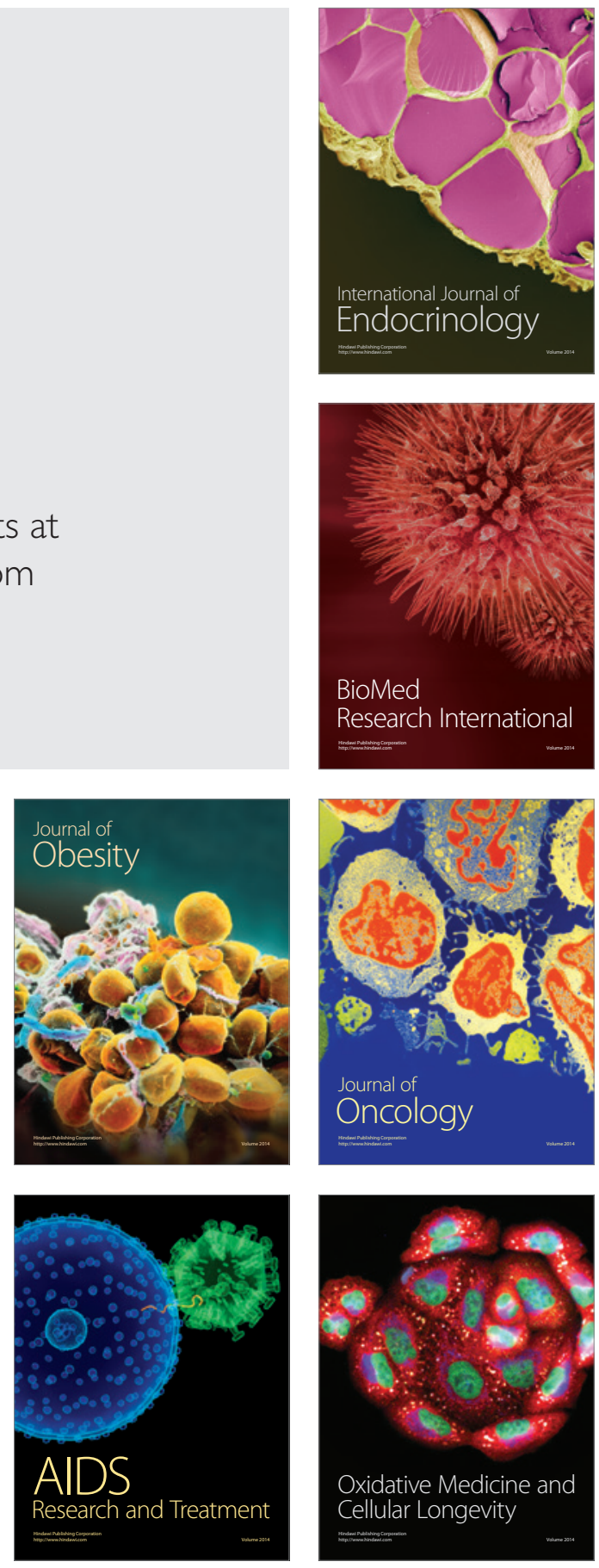